Peter Kirschner

Abteilung Unfall- und Wiederherstellungschirurgie, St.-Vincenz- und Elisabeth-Hospital, Mainz

\title{
zur Unfallmedizinischen Tagung, Leipzig 2001
}

Als amtierender Präsident der Deutschen Gesellschaft für Unfallchirurgie möchte ich ihnen die herzlichen Grüße unserer wissenschaftlichen Fachgesellschaft überbringen und dabei die Gelegenheit nutzen, in einer Zeit ständiger Neuerungen im Gesundheitswesen bei enger werdenden Budgets auf die Wichtigkeit des vertrauensvollen Zusammenwirkens hinzuweisen.

Die deutsche Unfallchirurgie nimmt wegen ihrer allumfassenden Versorgung Unfallverletzter auf wissenschaftlich und technisch modernstem Niveau weltweit eine führende Stellung ein. Dies ist ein Verdienst unserer Fachgesellschaft, die die wissenschaftliche, praktische und interdisziplinäre Tätigkeit auf dem Gesamtgebiet der Unfallheilkunde und besonders der Unfallchirurgie gefördert hat.

Als ebenso hervorragend und einzigartig im internationalen Vergleich ist der Einfluss der gewerblichen Berufsgenossenschaften als Träger der gesetzlichen Unfallversicherung auf die Entwicklung der Unfallchirurgie anzusehen. Das berufsgenossenschaftliche Heilverfahren, nach welchem laut Gesetzestext die Wiederherstellung des Verletzten mit allen geeigneten Mitteln gefordert wird, setzt voraus, dass sich der Unfallversicherungsträger mit allen modernen und anerkannten Möglichkeiten der Behandlung und Wiederherstellung vertraut macht, für ihre Sicherstellung und Durchführung sorgt sowie auch Unfallverhütung betreibt.

Damit stellt das Heilverfahren nicht nur Grundlage, sondern auch Bindeglied zwischen den Trägern der gesetzlichen Unfallversicherungen und der Unfallchirurgie dar.

Des Weiteren muss erwähnt werden, dass von öffentlicher Seite nach dem 2. Weltkrieg, in einer Zeit der stür- mischen Entwicklung von Industrialisierung und Verkehr, im Hinblick auf die Schaffung traumatologischer Schwerpunkte in Klinik, Forschung und Lehre dem notwendigen Bedarf nicht ausreichend Rechnung getragen wurde.

Hier haben die gewerblichen Berufsgenossenschaften mit der Neugründung von Unfallkliniken beispielhaft eine Grundlage für die Entwicklung moderner Unfallchirurgie geschaffen.

Mit der Einrichtung unfallchirurgischer Lehrstühle durch die öffentliche Hand an deutschen Universitäten $z u$ Beginn der joer Jahre wurden dann die weitere Entwicklung der Unfallchirurgie nochmals beschleunigt und die $\mathrm{Ba}$ sis für Forschung und Lehre verbessert.

Hierbei haben 3 Faktoren den Fortschritt wesentlich beeinflusst:

1. Die kritische Erkenntnis, dass bisherige Behandlungsergebnisse nicht immer dem Anspruch der Patienten, aber auch der Ärzte genügten, führte durch systematische Erfassung von Fehlleistung und lückenloser Dokumentation zur Eliminierung von exogenen Noxen im Sinn einer Prophylaxe, wozu auch die Vorsorge und Präventionsmaßnahmen der Berufsgenossenschaften zählen. Exakte Diagnostik und sachgemäße Erstversorgung haben daneben gröBere Chancen für optimale Behandlungsergebnisse eröffnet. Wissenschaftliche Fachgesellschaft und Versicherungsträger sehen daher mit Recht in der Qualitätssicherung ein Instrument zur Optimierung sowohl der ärztlichen Tätigkeit als auch der Behandlungsergebnisse.

2. Der 2. Faktor für den Fortschritt, auch aus berufsgenossenschaft- licher Sicht, ist der Entwicklungsstand der Techniken im Hinblick auf Instrumentation und $O P$ Ausstattung, diagnostische Gerätschaften, Ausbau der Anästhesie und Intensivmedizin sowie des Notfall- und Rettungswesens.

3. Der 3. Punkt beruht, wie auch in anderen Bereichen, auf den Erkenntnissen der Grundlagenforschung. Hier haben enge Verbindungen von berufsgenossenschaftlichen Unfallkliniken mit universitären Einrichtungen im klinischen und wissenschaftlichen Bereich produktive Forschungsarbeit geleistet.

Neben den klar gegliederten Aufgaben und Auflagen, in welchen sich wissenschaftliche Fachgesellschaft und Unfallversicherungsträger entwickelt haben und bewegen, war das Verhältnis zwischen Berufsgenossenschaft und Deutscher Gesellschaft für Unfallchirurgie immer von herausragenden Persönlichkeiten auf beiden Seiten getragen und von einer vertrauensvollen Zusammenarbeit geprägt.

An dieser Stelle möchte ich Herrn Dr. Sokoll zitieren, der in einem Referat "Quo vadis Unfallversicherung" Folgendes ausgeführt hat:

\section{„....dass die belegten Erfolge der Unfall- versicherungsträger in Heilbehandlung und Rehabilitation ganz maßgeblich auf die organisierte Kooperation mit der Un-}

\footnotetext{
๑) Springer-Verlag 2003

Prof.Dr. Peter Kirschner Abteilung Unfallund Wiederherstellungschirurgie, St.-Vincenz- und Elisabeth-Hospital, An der Goldgrube 11,55131 Mainz
} 


\section{Eröffnung}

fallmedizin zurückzuführen sind, dass wir - anders als dies in der Krankenversicherung zu sein scheint - uns nicht als Gegner verstehen, sondern dass wir im Interesse der Unfallverletzten - traditionell - eine gute Bündnispartnerschaft pflegen.

Es hat sich seit Begründung der besonderen berufsgenossenschaftlichen Heilverfahren eine Umgangskultur zwischen Ärzteschaft und den Unfallversicherungsträgern entwickelt, die Maßstäbe gesetzt hat, an denen sich auch andere Bereiche des Gesundheitswesens orientieren."

Die Maßgabe, den Unfallverletzten mit „allen geeigneten Mitteln“ wiederherzu- stellen, ist eine wesenskonforme Einstellung, die Unfallchirurgen und Unfallversicherungsträger in einer Art Grundauffassung verbindet.

Im Sinn unseres gemeinsamen Interesses zur Verbesserung der Behandlung Unfallverletzter ist dem aus meiner Sicht nichts hinzuzufügen.

Die Unfallchirurgie entwickelt sich weiter. Eine Zusammenführung mit der Orthopädie zu einem Fachgebiet Unfallund Orthopädische Chirurgie im Rahmen einer neuen Weiterbildungsordnung hat begonnen. Die Umstrukturierung der Deutschen Gesellschaft für Chirurgie in eine Dachgesellschaft für zunächst 8 chirurgische Spezialdisziplinen mit
- Viszeralchirurgie,

- Unfallchirurgie,

- Orthopädischer Chirurgie,

- Herzchirurgie,

- Thoraxchirurgie,

- Gefäßchirurgie,

- Plastischer Chirurgie und

- Allgemeiner Chirurgie

ist eingeleitet und stellt für diese Entwicklung eine Voraussetzung da. Der fachlichen Spezialisierung gehört die Zukunft.

\section{Verbrennungen}

\section{S. Hermann}

Klinik für Plastische und Handchirurgie/Brandverletztenzentrum, BG-Kliniken „Bergmannstrost”, Halle/Saale

\section{Ödembildung und Ödemresorption im Verbrennungsschock}

Autor hat kein Manuskript eingereicht

C.Schmidt

Abteilung für Plastische und Handchirurgie, Zentrum für Schwerbrandverletzte, Städtisches Krankenhaus München-Bogenhausen, München

\section{Strategie bei der operativen Primärbehandlung Schwerbrandverletzter}

Autor hat kein Manuskript eingereicht

E. Hoefter

Abteilung für Plastische und Handchirurgie, Zentrum für Schwerbrandverletzte, Städtisches Krankenhaus München-Bogenhausen, München

\section{Keratinozytenkultur und ihre klinische Bedeutung in der Behandllung Brandverletzter}

Autor hat kein Manuskript eingereicht 